\title{
Diagnóstico situacional dos Centros Especializados em Reabilitação (CER) e Oficinas Ortopédicas: Projeto CER-Brasil
}

\section{Situational diagnosis of Specialized Centers in Rehabilitation and Orthopedic Manufacturings: CERBrasil Project}

(D) Luciana Castaneda1, (D) Diego de Sousa Dantas², (D)Antônio Tadeu Ribeiro de Oliveira ${ }^{3}$, (DShamyr Sulyvan de Castro ${ }^{4}$

IInstituto Federal de Educação, Ciência e Tecnologia do Rio de Janeiro - IFR

2 Universidade Federal de Pernambuco - UFPE

${ }^{3}$ Instituto Brasileiro de Geografia e Estatística IBGE

${ }^{4}$ Universidade Federal do Ceará - UFC

\section{Correspondência}

Shamyr Sulyvan de Casto

E-mail: castross@ufc.br

Submetido: 23 Junho 2020

Aceito: 16 Fevereiro 2021

Projeto financiado

CNPq: Proc. 371129/2019-2

\section{Como citar}

Castaneda L, Dantas DS, Oliveira ATR, Castro SS. Diagnóstico situacional dos Centros Especializados em Reabilitação (CER) e Oficinas Ortopédicas: Projeto CER-Brasil. Acta Fisiatr. 2020;27(4):256-259.

DOI: 10.11606/issn.2317-0190.v27i4a171383

\section{RESUMO}

A atenção a pessoa com deficiência no Brasil vem ganhando destaque nos últimos anos, com a criação da Rede de Cuidados à Pessoa com Deficiência, bem como com iniciativas ministeriais para avaliação da situação atual da rede e dos serviços específicos em reabilitação. Nesse sentido, pretende-se por meio dessa comunicação breve, apresentar projeto de pesquisa nacional de avaliação da atenção especializada em reabilitação. 0 projeto intitulado CERBrasil objetiva realizar o diagnóstico situacional dos Centros Especializados em Reabilitação (CER) e Oficinas Ortopédicas. Trata-se de um estudo multicêntrico com a articulação nacional de pesquisadores das cinco regiões federativas brasileiras. Através de uma abordagem plurimetodológica, o projeto está sendo realizado em diferentes etapas, que incluem método Delphi, pesquisa documental, avaliação de serviços de saúde, e entrevista com gestores, profissionais e pacientes ligados aos CER em diferentes regiões do território brasileiro.

Palavras-chave: Pessoas com Deficiências, Serviços de Saúde, Centros de Reabilitação, Avaliação em Saúde

\section{ABSTRACT}

In Brazil, the attention to people with disabilities has increasing in recent years, with the creation of the Care Network for People with Disabilities, as well as with ministerial initiatives to assess the current situation of the network and specific services in rehabilitation. In this sense, it is intended, through this brief communication, to present the CERBrasil project that aims to carry out the situational diagnosis of Specialized Centers in Rehabilitation (SCR) and orthopaedics manufacturings. The CERBrasil project is a multicenter study with the national articulation of researchers from the five Brazilian federal regions. Through a multi-methodological approach, the project is being carried out in different stages, which include the Delphi method, documentary research, evaluation of health services, and interviews with managers, professionals and patients linked to SCR in different regions of the Brazilian territory.

Keywords: Disabled Persons, Health Services, Rehabilitation Centers, Health Evaluation 


\section{INTRODUÇÃO}

O interesse pela reabilitação e saúde das pessoas com deficiência, cresce no Brasil e no mundo, como consequência do número de pessoas com doenças crônicas, taxa de envelhecimento populacional e valorização da pessoa com deficiência. ${ }^{1}$ No Brasil, a Rede de Cuidados à Pessoa com Deficiência (RCPD), constitui-se como rede de atenção direciona às pessoas com deficiência e visa orientar e fortalecer o processo de regionalização e avançar em uma enorme lacuna de cuidado a saúde, a oferta de Reabilitação no Sistema Único de Saúde (SUS). De acordo com a configuração da RCPD, os Centros Especializados em Reabilitação (CER) são serviços reconhecidos pelo Ministério da Saúde como os prestadores dos cuidados em reabilitação. ${ }^{2,3}$

Com fins de elucidar o panorama de avanço da rede, bem como os entraves à sua implementação, no ano de 2018, o Ministério da Saúde lançou chamada pública para apoiar projetos de pesquisa que visassem contribuir para o desenvolvimento científico, tecnológico e a inovação na área de saúde da pessoa com deficiência. Foram aprovados na chamada pública cinco projetos.

O projeto CERBrasil - Avanços, Desafios e Operacionalização dos Centros Especializados em Reabilitação (CER), é um dos projetos aprovados na linha de análise dos serviços de reabilitação habilitados como CER em relação à adesão aos princípios e às diretrizes definidos nas normativas da RCPD.

Trata-se de uma proposta plurimetodológica, fruto da articulação nacional da Rede Brasileira de Pesquisa em Funcionalidade ( https://rbpf.ufc.br/pt/ ). Essa comunicação breve visa apresentar à comunidade acadêmica e aos leitores da revista, os objetivos e o desenho metodológico que irá permitir a geração de dados e informação sobre a situação dos CER, na perspectiva da gestão do cuidado. Prevê ainda atender solicitações da demanda do executivo federal para a identificação do cenário da oferta e efetividade dos serviços de CER e Oficinas Ortopédicas no Brasil.

\section{Descrição do projeto}

O Projeto CERBrasil, aprovado pelo comitê de Ética em Pesquisa sob no CAAE 19909119.3.0000.5268, possui a vertente da pesquisa e inovação e tem por objetivo geral caracterizar os CER e Oficinas Ortopédicas como agentes de fortalecimento a Integralidade do Sujeito tendo como alinhamento a RCPD. O projeto conta com a colaboração de pesquisadores e instituições de ensino e pesquisa de dez unidades federativas, representando as cinco regiões brasileiras.

Dentre os objetivos específicos e etapas do projeto destaca-se:

1) identificar a abrangência e caracterização da rede dos CER no país;

2) elaborar um modelo lógico da RCPCD, identificando o papel dos CER no mesmo;

3) propor uma matriz de Indicadores da Efetividade e da Qualidade do cuidado ofertados pelos CER;

4) mapear a qualidade do cuidado integral ofertados pelos CER com a perspectiva dos Sistemas de Informação em Saúde, Gestores, Profissionais, Usuários e Especialistas;
5) apontar a articulação dos CER com os demais pontos de atenção à saúde e outras políticas públicas, como de assistência social, previdência e trabalho e emprego.

0 projeto fundamenta-se nos modelos mais atuais para descrição/entendimento da deficiência humana, o modelo biopsicossocial e holístico compreendido na Classificação Internacional de Funcionalidade, Incapacidade e Saúde - CIF, 4,5 bem como nos processos de avaliação e planejamento em saúde $^{6,7}$ com produção de informações na perspectiva de diferentes atores da política pública de reabilitação no país, usuários, profissionais e gestores, com vistas a produção de indicadores do processo de trabalho e qualidade da assistência prestada.

Tendo como pressuposto a importância na construção e no rigor científico para o protocolo de pesquisa orientado a melhoria da Governança da política de Reabilitação no país, visa o CERBrasil, caracterizar por uma matriz de indicadores aspectos envolvidos na qualidade do serviço de reabilitação ofertado na rede SUS. Incorporar indicadores que reflitam a articulação dos CER e Oficinas Ortopédicas com os demais pontos da RCPCD também foram perspectivas incluídas. Sendo assim, cada etapa/objetivo específico, demanda a realização de métodos e atores específicos.

Para o levantamento da abrangência e caracterização da rede dos CER no país, realizou-se a avaliação ex-post da política pública de Reabilitação no país, a perspectiva dos gestores do executivo (federal, estadual e municipal), força de trabalhos dos CER e Oficinas Ortopédicas, dos usuários, da literatura e de um painel de especialistas intersetorial.

A elaboração do modelo lógico da $\mathrm{RCPD},{ }^{8}$ demandou a realização de grupos focais com gestores e profissionais do CER. Enquanto que a proposição de matriz de indicadores da Efetividade e da Qualidade do cuidado ofertados pelos CER e itens relevantes à caracterização dos serviços foi definida por técnica de grupo nominal com os pesquisadores do comitê científico.

Para caracterização do cuidado ofertado e a adesão aos princípios propostos pela RCPCD, será realizado inquérito com representatividade nacional nos CER e Oficinas Ortopédicas. $O$ inquérito envolverá coleta de dados in loco, por entrevistadores previamente treinados, com gestores, profissionais, usuários e coletas de prontuários.

A definição do desenho amostral levou em consideração o pequeno volume de estabelecimentos distribuídos pelo território nacional, que totalizam apenas 217 unidades de CER e 39 de Oficinas Ortopédicas, de acordo com base de dados disponibilizada pelo Ministério da Saúde.

Neste sentido, a adoção de amostragem probabilística fica inviabilizada, uma vez que, neste caso, o tamanho da amostra seria muito próximo ao total de CERs, eliminando uma das vantagens comparativas desse tipo de amostragem que é justamente tornar menos onerosa a realização do levantamento. Assim, sugere-se que o desenho amostral caminhe no sentido da extração de amostras intencionais, em dois estágios. A distribuição da quantidade de CER e oficinas por unidade da federação pode ser visualizada nas Tabelas 1 e 2 , respectivamente. 0 primeiro estágio constitui-se de estratos por Grandes Regiões, a partir dos quais foi selecionada uma amostra intencional de 50 estabelecimentos de CER e 10 de Oficinas Ortopédicas. 
Tabela 1. Distribuição da amostra do CERBRASIL nos serviços de Reabilitação por Região e Porte de serviço de Reabilitação habilitados pela RCPD $(n=35)$

\begin{tabular}{lccccc}
\hline \multicolumn{1}{c}{ Região } & $\begin{array}{c}\text { Modalidade } \\
\text { Única }\end{array}$ & CER II & CER III & CER IV & $\begin{array}{c}\text { Total/ } \\
\text { Amostra }\end{array}$ \\
\hline Sudeste & 1 & 13 & 7 & 2 & 22 \\
Nordeste & - & 6 & 3 & - & 9 \\
Norte & 1 & 3 & 1 & - & 5 \\
Centro-oeste & - & - & - & 1 & 1 \\
Sul & - & 1 & - & - & 1 \\
Total & 2 & 21 & 10 & 3 & 35 \\
\hline
\end{tabular}

Tabela 2. Distribuição da amostra do CERBRASIL nas Oficinas Ortopédicas por Região e Porte de serviço de Reabilitação habilitados pela RCPD $(n=9)$

\begin{tabular}{lccccc}
\hline \multicolumn{1}{c}{ Região } & $\begin{array}{c}\text { Modalidade } \\
\text { Única }\end{array}$ & CER II & CER III & CER IV & $\begin{array}{c}\text { Total/ } \\
\text { Amostra }\end{array}$ \\
\hline Sudeste & 1 & 4 & - & - & 5 \\
Nordeste & - & 1 & - & - & 1 \\
Norte & 1 & - & - & - & 1 \\
Centro-oeste & - & - & - & 1 & 1 \\
Sul & - & 1 & - & - & 1 \\
Total & 2 & 6 & 0 & 1 & 9 \\
\hline
\end{tabular}

Essa amostra foi distribuída proporcionalmente em cada um dos estratos, obedecendo-se a participação relativa das respectivas Unidades da Federação. A população de estudo no inquérito são os gestores dos estabelecimentos, ao menos três profissionais de saúde em cada CER e ao menos três usuários dos serviços. O segundo estágio da amostra foi estruturado de forma a possibilitar a investigação dos prontuários dos usuários em cada um dos estabelecimentos selecionados no primeiro estágio. A estratégia adotada visou privilegiar a seleção de prontuários conforme o tipo deficiência tratado em cada modalidade de CER, amostrado no primeiro estágio. Assim, em cada estabelecimento devem ser selecionados as seguintes quantidades de prontuários: CER II - 24 prontuários para cada tipo de deficiência; CER II - 16 prontuários para cada tipo de deficiência; CER IV - 12 prontuários para cada tipo de deficiência. Ao todo, nos 50 estabelecimentos selecionados, o tamanho total da amostra de prontuários será de 2.400 pacientes. Esses prontuários serão escolhidos de forma aleatória, respeitando-se o volume de prontuários por tipo de deficiência em cada CER.

A seleção dos prontuários levará em consideração o total de prontuário do arquivo dos últimos cinco anos. Para definição do intervalo de seleção será aplicada a fórmula: $I=N / n$, onde $\mathrm{N}$ se refere ao total de prontuários e $\mathrm{n}$ ao tamanho amostral estabelecido por tipo de deficiência. Tomando-se a parte inteira do resultado de I, será escolhido um primeiro prontuário aleatoriamente, e a partir daí soma-se o resultado de I para definir os demais elementos da amostra.

O instrumento piloto construído com base nos resultados das etapas 1 e 2 foi submetido a análise de validade de conteúdo por estudo Delphi, realizado por especialistas e membros do projeto no mês de junho de 2019.

Após rodada de deliberação, o instrumento de avaliação
CERBrasil foi elaborado e submetido a validação de conteúdo por gestores e profissionais de saúde ligados à reabilitação.

$O$ instrumento de avaliação a ser aplicado nos serviços possui quatro dimensões, I - Modulo Gestor, II - Modulo Profissionais de saúde, III - Modulo Usuário e IV - Modulo Prontuário. Os itens de avaliação dos módulos I e II foram baseados nos indicadores da Pesquisa Nacional de Avaliação dos Serviços de Saúde (PNASS) ${ }^{5}$ e nos resultados de modelo lógico elaborado a partir de Grupo Focal realizado com profissionais e gestores dos CER do estado do Rio Grande do Norte, em estudo que se encontra em fase final de execução.

Os itens do Módulo usuário são compostos por informações sociodemográficas, de Funcionalidade, de Qualidade de Vida Relacionada a Saúde além de satisfação do usuário. Os itens relativos à Funcionalidade serão abordados pelo WHODAS versão 12 itens $^{9}$ acrescidos de informações de Fatores Ambientais provenientes de estudo prévio realizado com a população brasileira. ${ }^{10} \mathrm{O}$ Módulo Prontuário apresenta duas subdimensões que tratam de desfechos de Gestão da Clínica dos sujeitos com informações sobre a utilização da Classificação Internacional de Funcionalidade (CIF), construção do Projeto Terapêutico Singular (PTS), utilização de escalas de avaliação e itinerário terapêutico.

A fim de diminuir e gerir os desafios inerentes à uma pesquisa multicêntrica, constitui-se um comitê gestor para a pesquisa, possibilitando uma construção democrática do projeto em tela, bem como do percurso metodológico e produtos, com decisão colegiada e debatida em reunião de consenso.

\section{CONCLUSÕES}

A pesquisa encontra-se em andamento e espera-se ao final produzir evidências científicas sobre a gestão do cuidado ofertada pelos centros especializados em reabilitação e panorama sobre a política pública brasileira de reabilitação. Além do caráter descritivo dos dados, espera-se contribuir para o processo de tomada de decisão e gestão em saúde, por meio do fornecimento de dados baseados em indicadores de efetividade do cuidado. Além disso, a incorporação de tecnologias baseadas no modelo biopsicossocial com amplo alcance são expectativas de desenvolvimento tecnológico que podem ser geradas a partir dos resultados do CERBrasil.

Os produtos de inovação propostos visam compor o desenvolvimento de Manual de Tecnologias Leves para suporte a construção dos Projetos Terapêuticos Singulares. Também está prevista a transferência de tecnologia para criação de observatório de política pública (OPP): observatório da Inclusão. O Manual de Tecnologias Leves e o OPP visam disseminar:

I - A utilização da CIF como uma tecnologia leve;

II - A criação de uma cultura de linguagem mais homogênea para descrição das consequências das condições de saúde;

III - A orientação do raciocínio de construção de programas terapêuticos individuais pela abordagem biopsicossocial;

IV - O arcabouço alfanumérico da CIF para suporte a sistemas de informação com dados da Funcionalidade.

\section{AGRADECIMENTO}

Ao Ministério da Saúde. 


\section{REFERÊNCIAS}

1. Heinemann AW, Feuerstein M, Frontera WR, Gard SA, Kaminsky LA, Negrini $S$, et al. Rehabilitation is a global health priority. Arch Phys Med Rehabil. 2020;101(4):728729. Doi: https://doi.org/10.1016/i.apmr.2019.08.468

2. Brasil. Ministério da Saúde. Portaria n.793, de 24 de abril de 2012. Institui a Rede de Cuidados à Pessoa com Deficiência no âmbito do Sistema Único de Saúde. Diario Oficial da Republica Federativa do Brasil, Brasilia (DF); 2012 Abr 25; Seção 1: 94-95.

3. Campos MF, Souza LA, Mendes V. A rede de cuidados do Sistema Único de Saúde à saúde das pessoas com deficiência. Interface (Botucatu). 2015;19(52):207-10. Doi: https://doi.org/10.1590/1807-57622014.0078

4. World Health Organization. International Classification of Functioning, Disability and Health (ICF). Geneva: WHO; 2001.

5. Jackson M. Models of disability and human rights: informing the improvement of built environment accessibility for people with disability at Neighborhood Scale? Laws. 2018;7(1):10. Doi: https://doi.org/10.3390/laws7010010
6. Hartz ZMA. Avaliação em Saúde: dos modelos conceituais à prática na análise da implantação de programas. Rio de Janeiro: $\quad$ FIOCRUZ; $1997 . \quad$ Doi: https://doi.org/10.7476/9788575414033

7. Brasil. Ministério da Saúde. Manual de planejamento no SUS. Brasília: Ministério da Saúde, Fundação Oswaldo Cruz; 2016. [Série Articulação Interfederativa, 4].

8. Cassiolato M, Gueresi S. Como elaborar Modelo Lógico: roteiro para formular programas e organizar avaliação. Brasília: IPEA; 2010 [Nota Técnica, 6].

9. Silveira C, Parpinelli MA, Pacagnella RC, Camargo RS, Costa ML, Zanardi DM, et al. Adaptação transcultural da Escala de Avaliação de Incapacidades da Organização Mundial de Saúde (WHODAS 2.0) para o Português. Rev Assoc Med Bras. 2013;59(3):234-40. Doi: http://dx.doi.org/10.1016/j.ramb.2012.11.005

10. Castro SS, Cieza A, Cesar CL. Problems with accessibility to health services by persons with disabilities in São Paulo, Brazil. Disabil Rehabil. 2011;33(17-18):1693-8. Doi: http://dx.doi.org/10.3109/09638288.2010.541542 